\title{
3 Zur Verwendung der perfektiven Präsensform im heutigen Tschechisch
}

\subsection{Einführung}

In der Forschung zum slawischen Aspekt wurde bis heute kaum den Präferenzen verschiedener Formen Aufmerksamkeit gewidmet, die sich auf die tatsächliche Verwendung einer bestimmten morphologischen Verbalform beziehen. ${ }^{48}$ Die in Kapitel 5, Kapitel 8 und Schmiedtová/von Stutterheim/Carroll (2011) dargestellten Ergebnisse zeigen, dass Muttersprachler des Tschechischen und des Russischen trotz der Ähnlichkeiten in den zugrundeliegenden Aspektsystemen unterschiedliche Präferenzen für verschiedene morphologische Verbalformen in der Darstellung eines bestimmten Situationstyps zeigen.

Tschechische Muttersprachler verwenden präfigierte Verben, die vom grammatischen Aspekt her meistens perfektiv sind, überwiegend für Situationen, in denen es sich um den Nachzustand ${ }^{49}$ einer Handlung handelt, also beispielsweise für eine Situation, in der eine Frau ein Glas Wasser ganz austrinkt (man sieht sie das Glas heben, dann das Wasser trinken und schließlich das Glas wieder auf der Tischoberfläche abstellen). Eine charakteristische Versprachlichung einer solchen Szene im Tschechischen ist: tsch. Paní vy-pila (perfektive Vergangenheitsform) - dt. Eine Frau trank ein Glas Wasser aus (siehe auch Kap. 1-2 für die Klärung des Aspektbegriffs).

Die präfigierte Form lässt sich im Tschechischen auch für Bewegungsereignisse vom Typ [-END] belegen: tsch. Pán ve-šel do domu (perfektive Vergangenheitsform) - dt. Ein Mann ging ins Haus rein. In Situationen dieses Typs wird eine Bewegung gezeigt, in der ein potenziell zu enkodierendes Ziel (z.B. ein Gebäude am Ende eines Weges, auf dem sich zwei Menschen bewegen) als nicht erreicht [-END] dargestellt wird. Mit anderen Worten, wenn ein solcher Endpunkt enkodiert wird, muss dieser zuvor inferiert werden. In russischen Daten kommen diese Formen nur in jenen Szenen vor, in denen eine Bewegung von Punkt A zu Punkt B als erreicht gezeigt wird [+END].

48 Eine Ausnahme stellt die auf qualitativer Analyse basierte Arbeit von Petruchina (2000) dar, in der die Aspektkategorie sowie ihre aspektuellen Werte im Russischen mit der im Bulgarischen, Polnischen, Slowakischen und Tschechischen verglichen wird.

49 Ein Nachzustand ist der Teil der Handlung, zu dem eine Zustandsveränderung führt. Zum Beispiel bei dem Prädikat ein Glas Wasser austrinken ist der Nachzustand ein Glas Wasser ausgetrunken haben. 
Eine andere markierte Aspektform, die sekundären Imperfektiva, die vom grammatischen Aspekt her immer imperfektiv sind, werden von tschechischen Sprechern für die Beschreibung von Einzelereignissen eher selten gebraucht (etwa bei 10\% aller befragten Sprecher). Dagegen kommen sie in russischen Daten sehr häufig vor und zwar vorwiegend in Szenen mit Ereignissen, in denen das Erreichen des Nachzustands zu erwarten ist bzw. dargestellt wird (z.B. die oben erwähnte Szene 'eine Frau trinkt ein Glas Wasser aus'). Eine detaillierte Darstellung dieser Zusammenhänge ist in Kapitel $8 \mathrm{zu}$ finden.

Der markanteste Unterschied in der Anwendung der Aspektformen bezieht sich jedoch auf den Gebrauch der präfigierten perfektiven Präsensformen, die im Tschechischen auch für die Online-Darstellung der hier und jetzt ablaufenden Ereignisse gebraucht werden. Die tschechischen Probanden gebrauchen für die Beschreibung der oben beschriebenen Szene die präfigierte perfektive Präsensform, während der Videoclip noch läuft (tsch. Paní vy-pije sklenici vody (perfektive Präsensform) - dt. Eine Frau trinkt ein Glas Wasser aus). Manchmal wird in diesem Kontext das temporale Adverb ted', právě (dt. jetzt) verwendet. In den tschechischen Grammatiken ist diese Verwendung der perfektiven Präsensformen als nicht zulässig beschrieben (vgl. Cvrček et al. (Hgg.) 2010: 245; Komárek et al. (Hgg.) 1986: 17950). Im Russischen werden präfigierte perfektive Präsensformen in Verbindung mit der Hier-und-jetzt-Bedeutung nie verwendet. Diese Aspektform hat im Russischen immer eine Zukunftsbedeutung. ${ }^{51}$

In Bezug auf den Aspektgebrauch in den beiden slawischen Sprachen können also signifikante Unterschiede festgestellt werden: Anders als im Russischen werden im Tschechischen präfigierte perfektive Verbformen für die Versprachlichung von Ereignissen mit Nachzustand verwendet; in manchen Fällen kommt

50 „Dokonavá slovesa nemohou vyjadřovat děj probíhající $v$ přítomnosti, ale pouze to, že se děj již uskutečnil, nebo ještě nikoliv (koupil jsem, koupím knihu).“ Deutsche Übersetzung: Perfektive Verben können keinen in der Gegenwart verlaufenden Vorgang ausdrücken, sondern nur einen Vorgang, der bereits geschehen ist oder noch geschehen wird (ich kaufe, ich kaufte ein Buch) (vgl. Komárek et al. (Hgg.) 1986: 179).

51 Die verschiedenen Funktionen und Verwendungsbedingungen der perfektiven Präsensform (PfP-Form) im Russischen wurden anhand von Übersetzungsdaten (Russisch-Deutsch und Russisch-Französisch) von Rathmayr (1976) ausgearbeitet. Neben den vielen fakultativen Verwendungsweisen der PfP-Form (z.B. Bezeichnung einer atemporalen, modalen und/oder stilistischexpressiven Handlung) bezeichnet sie, wenn obligatorisch verwendet, eine konkret-futurische non-modale Handlung. Unter dem Begriff konkret-futurische sind sowohl von der Gegenwart aus gesehen zukünftige als auch zum Zeitpunkt in der Vergangenheit nachzeitige Handlungen zu verstehen (ebd.: 169f.). Bei den vielseitigen Funktionen und Verwendungsmöglichkeiten der russischen PfP-Form wurde für das Russische keine identifiziert, die in Verbindung mit der Hierund-jetzt-Interpretation steht. 
diese Form sogar in der Verbalisierung zielorientierter Bewegungsereignisse vor. Ganz besonders auffallend ist dabei der Gebrauch der perfektiven Präsensformen in Verbindung mit der Hier-und-jetzt-Bedeutung, der im Tschechischen durchaus möglich, im Russischen jedoch ganz ausgeschlossen ist (siehe auch Kap. 1, Abschn. 1.1).

Aus Sicht der Forschung stellt sich die Frage, inwiefern die Befunde zur Verwendung der perfektiven Präsensform (PfP-Form) im Tschechischen als Phänomen des Sprachwandels oder als kontextabhängige individuelle 'Sonderverwendung' interpretiert werden können? Insbesondere geht es hier um die folgenden Fragen:

1. Wird die PfP-Form mit Hier-und-jetzt-Interpretation auch unter Verwendung eines anderen Testparadigmas gebraucht?

2. Ist die Benutzung der PfP-Form mit Hier-und-jetzt-Interpretation regional oder dialektal gebunden?

3. Hängt die Verwendung der PfP-Form mit Hier-und-jetzt-Interpretation von der Verbalklasse ab, der das gegebene Verb angehört?

4. Welche Implikationen hat die Existenz der PfP-Form mit Hier-und-jetzt-Interpretation für das Aspektsystem des Tschechischen?

Um diesen Fragen nachzugehen, wurde für das Tschechische eine umfangreiche empirische Studie zu Aspektpräferenzen in Hier-und-jetzt-Kontexten durchgeführt. Die ersten drei Fragestellungen werden im Abschnitt 3.4 (Ergebnisse) behandelt, auf Frage 4 wird im vorletzten Abschnitt (Fazit und Diskussion) eingegangen. Im Folgenden wird die Studie vorgestellt.

\subsection{Design der vorliegenden Studie}

Zur Untersuchung der oben genannten Fragestellungen wurde ein Präferenztest entwickelt. In Form eines Fragebogens ${ }^{52}$ wurden den Probanden 35 kurze auf Tschechisch verfasste Szenarien als gedruckter Text präsentiert. Am Anfang jedes Fragebogens stand eine ausführliche Anweisung (siehe Anhang A für den genauen Wortlaut). Die Aufgabe der Probanden bestand darin, die Szenarien zu lesen und nach jedem Szenario aus fünf verschiedenen Aspekt-Tempus-Alternativen diejenige auszuwählen, die das präsentierte Szenario am besten wiedergibt. Im zweiten Schritt sollten die Probanden die zweitbeste Alternative wählen und auf einer Skala von eins (sehr gut) bis fünf (nicht akzeptable) angeben, in wel-

52 Die vollständige Version des Fragebogens ist dem Anhang A zu entnehmen. 
chem Verhältnis die zweitbeste Alternative zur besten Alternative steht. In diesem Kapitel liegt der Fokus auf der Auswertung der Daten, die sich auf die Wahl der erstbesten Alternative bezieht.

Die Probanden wurden in unterschiedlich großen Gruppen getestet. ${ }^{53}$ Ihnen stand ausreichend Zeit zur Verfügung, um den achtseitigen Fragebogen auszufüllen. ${ }^{54}$ Am Ende jedes Fragebogens wurden Informationen über Alter und Geschlecht sowie über Fremdsprachenkenntnisse abgefragt. Testsprache war Tschechisch.

Parallel zu dieser Studie wurde zusätzlich eine Studie zum Russischen durchgeführt. Der im Russischen verwendete Fragebogen war eine Übersetzung ${ }^{55}$ der tschechischen Vorlage und wurde den Probanden elektronisch zugeschickt. Die vorliegende Studie hat das Tschechische im Fokus und zieht das Russische lediglich zum Vergleich heran. Daher wird hier nur das für den tschechischen Fragebogen relevante experimentelle Design detailliert erläutert. Auf das Russische wird in den Abschnitten Probanden (siehe Abschn. 3 dieses Kapitels) sowie Ergebnisse (siehe Abschn. 4 dieses Kapitels) Bezug genommen.

Das folgende Beispiel (17) stammt aus dem Fragebogen (zuerst auf Tschechisch, dann die deutsche Übersetzung). In dem Beispiel wird das Prädikat trinken (tsch. pít) mit Absicht vermieden (siehe unten für weitere Erläuterungen):

tsch.

Představte si, že jste v kavárně a někdo vedle u stolečku má v ruce kávu. S pitím je skoro hotový. Jak to s největší pravděpodobností vyjádřite?

dt.

Stellen Sie sich eine Situation vor, in der Sie in einem Café sind und jemand am Nachbartisch in der Hand einen Kaffee hält. Die Person ist mit dem Trinken fast fertig. Wie drücken Sie mit höchster Wahrscheinlichkeit eine solche Situation aus?

53 Die Untersuchung wurde an mehreren tschechischen Gymnasien und Universitäten durchgeführt. Eine an einem Gymnasium getestete Gruppe umfasste etwa 30 Schüler, die an den Universitäten getesteten Gruppen waren in der Regel etwas kleiner - zwischen 10 und 15 Personen. Die Erhebungen wurden von fünf verschiedenen Personen durchgeführt, die alle den gleichen Anweisungen folgten, so dass eine formale Vergleichbarkeit der erhobenen Daten gewährleistet ist.

54 Das Ausfüllen eines Fragebogens hat durchschnittlich 20 Minuten in Anspruch genommen. 55 Ein großer Dank geht an Elena Albers für ihre Hilfe bei der Übersetzung des tschechischen Fragebogens ins Russische. 
Die Beschreibung des Szenarios war durchgehend in Präsensform gehalten - sie ist also unmissverständlich im Hier-und-jetzt-Kontext eingebettet. Alle verwendeten Verben waren imperfektive Simplex-Formen (d.h. keine präfigierten oder suffigierten Verben). Dabei handelte es sich immer um andere Verben als die, die in den Aspekt-Tempus-Alternativen vorkamen. ${ }^{56}$ Diese Maßnahme dient dazu, eine Beeinflussung der Probanden bei der Wahl der Aspekt-Tempus-Formen durch die in der Beschreibung erwähnten Verben zu vermeiden.

Der Text fing stets mit dem Satz Stellen Sie sich die Situation vor, ... an und endete mit der Frage Wie drücken Sie mit höchster Wahrscheinlichkeit eine solche Situation aus? Abgesehen von diesen zwei Standardsätzen beinhaltete die Beschreibung maximal zwei weitere Sätze.

Die auf die Beschreibungen der Szenarios folgenden Alternativen bestanden aus diesen fünf Aspekt-Tempus-Formen ${ }^{57}$ (Beispiel (18a)):

(18a) tsch. a. Někdo vedle u stolečku do-pije kávu. Perfektiv Präsens

b. Někdo vedle u stolečku do-pi-l kávu. Perfektiv Vergangenheit

c. Někdo vedle u stolečku pi-l kávu. Imperfektive Simplex-

Form Vergangenheit

d. Někdo vedle u stolečku pije kávu. Imperfektive SimplexForm Präsens

e. Někdo vedle u stolečku do-píjí kávu. Sekundäres Imperfektiv Präsens

Da es im Deutschen keine entsprechenden Aspektformen gibt, sind die folgenden Übersetzungen (18b) nur approximativ.

(18b) a. Jemand am Nachbartisch trinkt einen Kaffee aus.

b. Jemand am Nachbartisch trank einen Kaffee aus.

56 In wenigen Szenarien war die Verwendung der Verbalnomina - in Simplex-Form - aus Kohärenzgründen unumgänglich: z.B. tsch. sázení stromů ('das Pflanzen der Bäume’) oder tsch. pití kávy ('das Trinken des Kaffees').

57 Die erste Fassung des Fragbogens umfasste eine sechste Aspekt-Tempus-Form, nämlich das sekundäre Imperfektiv in der Vergangenheit. In der Pilotphase hat sich jedoch herausgestellt, dass die Wahl aus sechs verschiedenen Alternativen für die Probanden recht schwierig war, was u.a. zu einer deutlichen Verlangsamung führte. Da diese Untersuchung nicht auf den Unterschied in der temporalen Verwendung des sekundären Imperfektivs fokussiert war und da frühere Untersuchungen (z.B. Schmiedtová/von Stutterheim/Carroll 2011) zeigten, dass sie im Hierund-jetzt-Kontext nicht gebraucht wird, wurde zugunsten des gesamten Experimentablaufs auf diese Form verzichtet. 
c. Jemand am Nachbartisch trank einen Kaffee.

d. Jemand am Nachbartisch trinkt einen Kaffee.

e. Jemand am Nachbartisch ist dabei, einen Kaffee auszutrinken (im Sinne von fertig trinken).

Im Hinblick auf die fünf Aspekt-Tempus-Formen soll angemerkt werden, dass die perfektive Präsensform - z.B. tsch. do-pit; dt. fertig trinken - sowie die perfektive Vergangenheitsform - z.B. tsch. do-pi-l; dt. er hat fertig getrunken - mit verschiedenen präfigierten Verben gebildet wurden (aber nicht etwa mit perfektiven Simplex-Formen vom Typ tsch. dát - dt. geben). Die Szenarien wurden bezüglich des Geschlechts des Protagonisten so variiert, dass die Verteilung der Genusmarkierung am Partizip der imperfektiven Simplex-Vergangenheits- und Präsensform (- $l$ für maskulin; -la für feminin) vergleichbar war (16 Maskulina; 12 Feminina). Darüber hinaus gab es zwei Szenarien im Neutrum (das Partizip endet auf -lo) sowie fünf Szenarien im Plural (das Partizip endet auf -li oder -ly je nach Genus und Belebtheit).

Die Reihenfolge der dargebotenen Aspekt-Tempus-Formen wurde randomisiert, so dass jede der getesteten Situationen eine andere Reihenfolge der Formen beinhaltete. Der einzige Unterschied zwischen den Alternativen (a) bis (e) bestand in der Aspekt-Tempus-Form. Alle anderen Komponenten - wie die Wortstellung, das verwendete Verb - waren identisch.

Insgesamt wurden 35 kurze Szenarien getestet, die sich in 15 kritische Stimuli und 20 Kontrollen gliederten. Die Kontrollen stellten Bewegungsereignisse dar, wie z.B. gehen, laufen, fahren, trampeln, schwimmen, sich nähern etc. Manche Bewegungsverben wurden wiederholt verwendet, allerdings immer mit verschiedenen Präfixen.

Die kritischen Stimuli stellten verschiedene Situationen mit Zustandsveränderung dar (z.B. etwas austrinken, etwas ablecken, etwas aufwärmen). Bei den für die kritischen Stimuli verwendeten Verben wurde auf die Zugehörigkeit zu den Konjugationsklassen geachtet, in die die meisten tschechischen Verben eingeteilt werden. ${ }^{58}$ Das Tschechische unterscheidet fünf Konjugationsklassen, die sich weiter in verschiedene Muster differenzieren lassen. Die Einordnung in eine Konjugationsklasse richtet sich nach der morphologischen Endung der Verben in der 3. Person Singular Präsens. Andere Eigenschaften wie beispiels-

58 Die sogenannten regelmäßigen Verben, zu denen die meisten tschechischen Verben gehören, lassen sich einer der Konjugationsklassen zuordnen. Eine kleine Gruppe von hochfrequenten Verben lässt sich dieser Klassifikation nicht zuordnen. Dies sind die sogenannten unregelmäßigen Verben (vgl. auch Komárek et al. (Hgg.) 1986; Cvrček et al. (Hgg.) 2010). 
weise die Semantik oder der Aspekt der Verben werden bei dieser Einteilung nicht berücksichtigt.

Da diese Klassifikation die überwiegende Zahl der tschechischen Verben klar einordnet, gilt es zu überprüfen, ob die Konjugationsklasse des Verbs mit der Wahl der PfP-Form in Verbindung steht. In Tabelle 4 sind die Konjugationsklassen zusammengefasst:

Tab. 4: Verbklassen im Tschechischen

\begin{tabular}{llll}
\hline Verbklasse & $\begin{array}{l}\text { Endung } \\
\text { (3.Sg.Mask.Präs) }\end{array}$ & Beispiel & deutsche Übersetzung \\
\hline I. Klasse & $-e$ & syp-e & 'er schüttet' \\
\hline II. Klasse & $-n e$ & tisk-ne & 'er druckt/drückt' \\
\hline III. Klasse & $-j e$ & pi-je & 'er trinkt' \\
\hline IV. Klasse & $-i ́$ & sed-í & 'er sitzt' \\
\hline V. Klasse & $-a ́$ & děl-á & 'er macht' \\
\hline
\end{tabular}

Für jede Verbklasse wurden drei verschiedene Verben ausgewählt. Die gewählten Verben waren alle hochfrequent und mit allen fünf Aspekt-Tempus-Formen kombinierbar (siehe Anhang F, Tab. 1 - für die Übersicht verwendeter Verben in Kombination mit den entsprechenden Aspekt-Tempus-Formen). ${ }^{59}$ Die Anordnung der kritischen und der Kontrollstimuli wurde randomisiert.

Der Fragebogen wurde in einer Pilotphase mit insgesamt 10 tschechischen Muttersprachlern getestet, die teilweise über Fachkompetenz in der Linguistik verfügten. Auf Basis der Antworten sowie der Kommentare der Probanden wurden minimale Anpassungen des Fragebogens durchgeführt. Die Änderungen betrafen die Anzahl der Aspekt-Tempus-Alternativen (siehe Fußnote 8) sowie stilistische und formale Aspekte.

59 Die Kombinierbarkeit der Verben mit den entsprechenden Aspekt-Tempus-Formen wurde von der Autorin und zusätzlich von zwei Linguisten mit Tschechisch als Muttersprache überprüft. 


\subsection{Probanden}

Insgesamt haben an dieser Studie 256 Probanden teilgenommen. ${ }^{60}$ Sie kamen aus vier verschiedenen Regionen Tschechiens: ${ }^{61}$ Mittelböhmen - Praha und Umgebung; Nordböhmen - Ústí nad Labem; Ostböhmen - Hradec Králové; Nordmähren - Ostrava und Třinec. ${ }^{62}$ In Tabelle 5 sind alle relevanten Informationen bezüglich der Probanden zusammengefasst:

Tab. 5: Übersicht - Probanden

\begin{tabular}{lccclll}
\hline Ort & $\mathbf{N}^{63}$ & $\begin{array}{l}\text { Geschlecht } \\
\text { weiblich }\end{array}$ & männlich & $\begin{array}{l}\text { Alters- } \\
\text { durchschnitt }\end{array}$ & $\begin{array}{l}\text { Alters- } \\
\text { spanne }\end{array}$ & Region \\
\hline $\begin{array}{l}\text { Praha u. } \\
\text { Umgebung }\end{array}$ & 79 & 57 & 22 & 22 Jahre & $19-30$ & $\begin{array}{l}\text { Mittel- } \\
\text { böhmen }\end{array}$ \\
\hline Hradec Králové & 57 & 31 & 26 & 17,6 Jahre & $17-20$ & Ostböhmen \\
\hline Ústí nad Labem & 25 & 21 & 4 & 20,9 Jahre & $19-24$ & Nordböhmen \\
\hline Třinec & 56 & 39 & 17 & 16,1 Jahre & $15-17$ & Nordmähren \\
\hline Ostrava & 40 & 33 & 7 & 19,9 Jahre & $19-26$ & Nordmähren \\
\hline Total & 256 & 181 & 75 & 19,3 Jahre & $17-30$ & \\
\hline
\end{tabular}

Der Tabelle 5 lässt sich entnehmen, dass die an der Studie teilnehmenden Probanden im Durchschnitt 19,3 Jahre alt waren. Dabei waren die jüngsten 17 und die ältesten Teilnehmer 30 Jahre alt. In allen Gruppen gab es mehr weibliche als

60 Ich möchte mich ganz herzlich bei den folgenden Kollegen/innen für die Unterstützung bei der Erhebung der tschechischen Daten bedanken (in alphabetischer Reihenfolge): Ilona Dvořáková (Hradec Králové), Andrea Germann (Třinec), Alena Hůrková (Hradec Králové), Jan Chromý (Praha), Martin Lachout (Ústí nad Labem, Ostrava), Eva Lehečková (Praha), Věra Pastorková (Třinec).

61 Es wäre sicherlich wünschenswert, auch aus der südmährischen Region Daten zu haben. Leider ist es der Autorin trotz mehrerer Anläufe nicht gelungen, Daten aus dieser Region zu gewinnen. Zugleich sollte betont werden, dass es angesichts der Ergebnisse der vorliegenden Studie plausibel anzunehmen ist, dass Sprecher aus Südmähren vergleichbare Präferenzen aufzeigen wie Sprecher aus den übrigen tschechischen Regionen.

62 Obwohl Ostrava und Třinec der gleichen Region angehören, werden in Anbetracht der dialektalen Ausprägung dieser beiden Varietäten die Ergebnisse der Datenanalysen separat präsentiert.

$63 \mathrm{~N}$ gibt die Gesamtzahl der Probanden an. 
männliche Personen. Alle Probanden waren tschechische Muttersprachler. Sie wuchsen in einer tschechischen Umgebung mit Tschechisch sprechenden Eltern auf. Alle Teilnehmer verfügten über Fremdsprachenkenntnisse (bei allen Probanden Englisch und/oder Deutsch, bei manchen auch Französisch oder Spanisch).

Die Daten in Hradec Králové sowie in Třinec wurden an den dortigen Gymnasien erhoben. Der Fragebogen wurde den Schülern zu Beginn einer Unterrichtsstunde als Übungsaufgabe präsentiert. Alle teilnehmenden Schüler kamen aus der jeweiligen Region und befanden sich zum Zeitpunkt der Datenerhebung in der letzten gymnasialen Klasse (Abiturklasse). In den übrigen Orten - Ostrava, Praha und Ústí nad Labem - fand die Datenerhebung an der jeweiligen Universität im Rahmen eines linguistischen Seminars statt. Die Studierenden, die in Ostrava und Ústí nad Labem aufgenommen wurden, stammten auch aus diesen Regionen. In dem Datenset, das in Prag erhoben wurde, waren 21 Studierende dabei, die nicht aus Prag, sondern aus anderen Regionen Tschechiens (vorwiegend aus Südmähren und Südböhmen) stammten. ${ }^{64}$ Da die durchgeführte Analyse ergab, dass sich die Antworten dieser Studierenden nicht bedeutend von den Antworten der aus Prag stammenden Studierenden unterschieden, wurden die Daten der 21 nicht aus Prag stammenden Teilnehmer zu den Prager Daten gezählt.

An der parallel durchgeführten Studie zur Verwendung der perfektiven Präsensform im Russischen haben insgesamt 35 Probanden teilgenommen. Von diesen Probanden waren 23 weiblich und 12 männlich. Das Durchschnittsalter betrug 24,5 Jahre (Altersspanne 18-31). Alle Probanden waren Studenten oder hatten bereits einen akademischen Abschluss und waren erwerbstätig. Sie haben Russisch als Muttersprache erlernt und wuchsen in russischer Umgebung mit russisch sprechenden Eltern auf. Alle bis auf zwei Probanden verfügten über Englisch- und/oder Deutschkenntnisse. Die Fremdsprache wurde erst im Schulalter erlernt.

Das tschechische und das russische Datenset sind also bezüglich der Variablen Alter, sozioökonomischer Hintergrund sowie Geschlechtsverteilung vergleichbar. Im Gegensatz zu den tschechischen wurden die russischen Daten elektronisch erhoben. ${ }^{65}$ Das heißt, die Teilnehmer bekamen den Fragebogen per

64 Die Karls-Universität in Prag hat unter den tschechischen Universitäten einen besonderen Stellenwert und zieht auch viele Studierende an, die ursprünglich nicht aus der Hauptstadt kommen.

65 Vielen Dank an Elena Grimmer, Natela Karakeshisheva, Valentina Meuren, Julia Napolova, Elena Albers und Ekaterina Travina für die Hilfe bei der Erhebung der russischen Daten. 
E-Mail zugeschickt, füllten ihn am Computer aus und sendeten ihn per E-Mail wieder an die Autorin zurück. Dieses Verfahren war zwar weniger kontrollierbar als das Verfahren für die Datenerhebung im Tschechischen, dafür aber logistisch deutlich einfacher zu bewältigen und zügiger durchführbar. Wie schon erwähnt steht das Russische nicht im Fokus der vorliegenden Untersuchung, sondern dient dem Tschechischen zum Vergleich. Die Verwendung der beschriebenen abweichenden Erhebungsmethode erscheint daher vertretbar, da sie eine signifikante Beeinflussung der Ergebnisse nicht erwarten lässt. Dies gilt sinngemäß auch für die Anzahl der russischen Probanden ( $\mathrm{N}=35)$ : Die Datenanalyse hat gezeigt, dass die Unterschiede zwischen dem Tschechischen und dem Russischen bezüglich der Verwendung der PfP-Form in Präsenskontexten auch bei der im Vergleich kleineren Anzahl der russischen Probanden sehr deutlich waren.

\subsection{Ergebnisse}

Die hier dargestellten Ergebnisse basieren auf den Auszählungen der AspektTempus-Formen, die von den Probanden für die kritischen Stimuli als die erstbeste Beschreibung gewählt wurden. Zu Beginn sollen die in der Einführung formulierten Fragen wiederaufgenommen und anhand von Daten beantwortet werden:

Fragestellung 1: Wird die PfP-Form mit Hier-und-jetzt-Interpretation auch unter Verwendung eines anderen Testparadigmas gebraucht?

Abbildung 6 ist Grundlage für die Beantwortung der ersten und zweiten Fragestellung $\left(\mathrm{StAb}=\right.$ Standardabweichung $\left.{ }^{66}\right)$ :

66 Die Standardabweichung wurde pro Gruppe auf der Basis aller Vorkommnisse der PfP-Form über alle vier Verbklassen berechnet. 


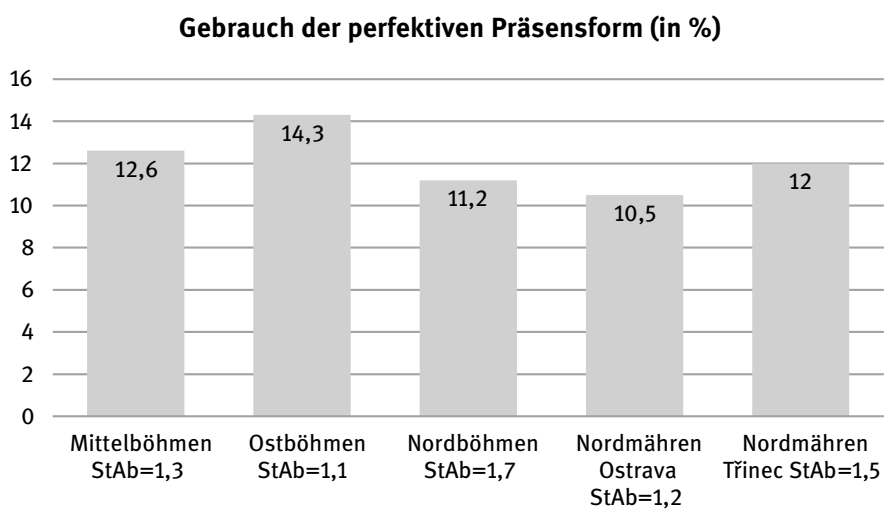

Abb. 6: Die Wahl der perfektiven Präsensform in Hier-und-jetzt-Kontexten von Probanden aus verschiedenen Regionen Tschechiens

Die Ergebnisse zu dem Gebrauch der perfektiven Präsensform im Tschechischen sind in Abbildung 7 in ihrer regionalen Verteilung dargestellt.

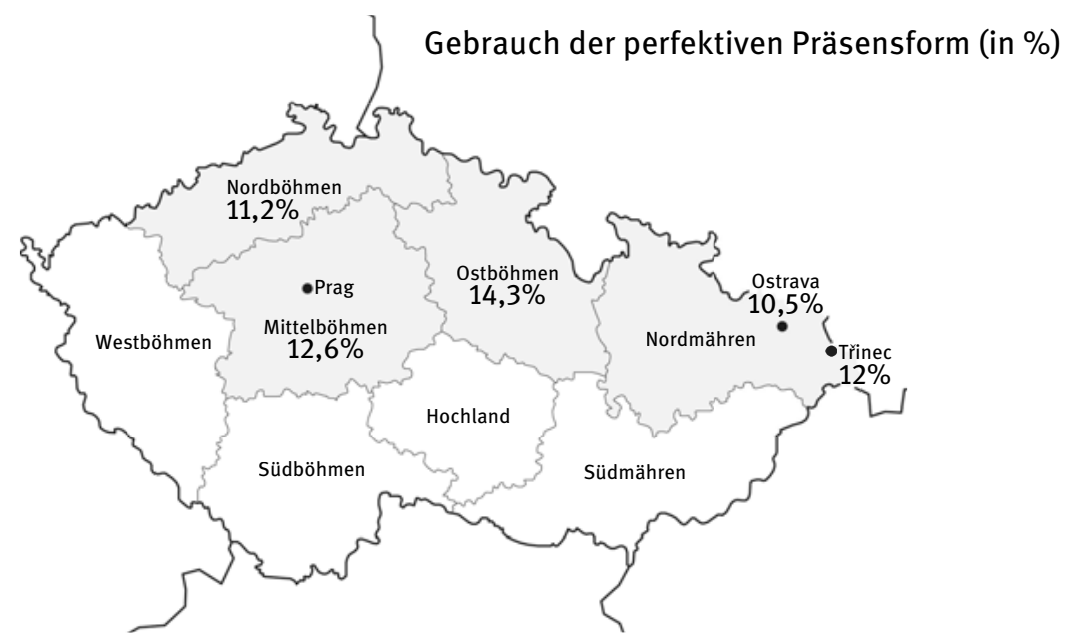

Abb. 7: Geografische Darstellung des Gebrauchs der perfektiven Präsensform in den untersuchten Regionen Tschechiens

In Bezug auf die erste Fragestellung kann festgehalten werden, dass die perfektive Präsensform in Verbindung mit der Hier-und-jetzt-Bedeutung nicht nur in Sprachproduktionsdaten, sondern auch im schriftlichen Präferenztest gewählt wird. Das 
heißt, der Präferenztest, der auf einer umfangreichen Datensammlung basiert, bestätigt die Befunde aus den Sprachproduktionsdaten (siehe Kap. 5 und 8).

Fragestellung 2: Ist die Benutzung der PfP-Form mit Hier-und-jetzt-Interpretation regional oder dialektal gebunden?

Hinsichtlich der zweiten Frage nach der Korrelation der Verwendung der PfP-Form von regionaler bzw. dialektaler Zugehörigkeit der Sprecher zeigt die Analyse (z-Test), dass es hinsichtlich der Verwendung der PfP-Präsensform in Hier-undjetzt-Kontexten keine statistisch relevanten Unterschiede gibt (Ostböhmen mit dem höchsten vs. Nordmähren-Ostrava mit dem niedrigsten Prozentwert: $\mathrm{z}=0,46, n s .{ }^{67}$ ). Die perfektive Präsensform in Hier-und-jetzt-Kontexten stellt für die Sprecher des Tschechischen also eine Alternative dar, die im aspektuellen System des Tschechischen zur Verfügung steht. Dies zeigt sich auch dadurch, dass diese Form konsistent (im Durchschnitt 12,1\%) und in vergleichbarem Maß in den verschiedenen Regionen Tschechiens im Hier-und-jetzt-Kontext verwendet wird (vergleichbare Standardabweichungen: Mittelböhmen =1,3; Ostböhmen =1,1; Nordböhmen =1,7; Normähren-Ostrava =1,2; Nordmähren-Třinec $=1,5$ ).

Bevor auf die Beantwortung der dritten Frage eingegangen wird, soll auf die Verteilung der anderen in dem Präferenztest getesteten Aspekt-Tempus-Formen eingegangen werden. Diese wird exemplarisch anhand der Daten aus Ostböhmen dargestellt (Abb. 8 - Angaben in Prozent):

Ostböhmen-Verteilung der Aspekt-Tempus-Formen

$(\mathrm{N}=855)$

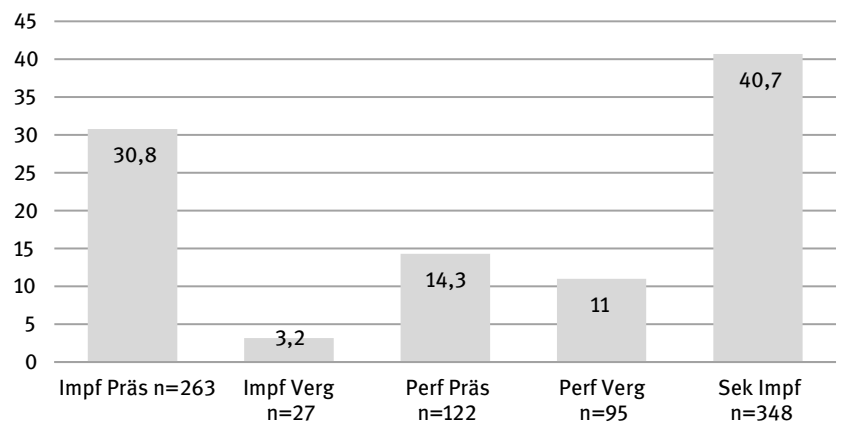

Abb. 8: Die Wahl der verschiedenen Aspekt-Tempus-Formen in Hier-und-jetzt-Kontexten von Probanden in Ostböhmen ( $\mathrm{N}=$ alle relevanten Datenpunkte (kritische Stimuli $\times$ Probandenzahl; $\mathrm{n}=$ absolute Zahl der verwendeten Aspekt-Tempus-Form)

67 Die Abkürzung ns steht für statistisch nicht signifikant. 
Aus der statistischen Auswertung wird deutlich, dass die Sprecher die imperfektiven Simplex- sowie die sekundären imperfektiven Präsensformen bevorzugt verwenden. Der Unterschied im Gebrauch des sekundären Imperfektivs ist gegenüber der Simplex-Form statistisch relevant $\left(\chi^{2}(1)=8,63, p<.05\right)$. Die häufige Wahl der präsentischen Formen, einschließend der perfektiven Präsensform, ist in Anbetracht der Einbettung der getesteten Szenarien in den Hier-und-jetzt-Kontext zu erwarten.

Die Präferenz für sekundäre Imperfektiva lässt sich auf die in den Szenarien dargebotenen Situationen zurückführen: Es wurden stets Handlungen mit Zustandsveränderung präsentiert. Gleichzeitig wurde betont, dass sich die Handlung ihrem Ende nähert. Für die Enkodierung solcher Situationen ist das sekundäre Imperfektiv geeignet.

Erwähnenswert ist der Gebrauch der perfektiven Vergangenheitsform, deren prozentualer Anteil trotz der Einbettung der Szenen ins Präsens relativ hoch ist. Der Unterschied zur imperfektiven Vergangenheitsform ist signifikant $\left(\chi^{2}(1)=34,32, p<.05\right)$. Diese Ergebnisse stehen im Einklang mit den Sprachproduktionsdaten: Muttersprachler des Tschechischen haben die perfektive Vergangenheitsform auch für die Online-Darstellung von Ereignissen mit qualitativem Nachzustand sowie von Bewegungsereignissen gebraucht (vgl. z.B. Schmiedtová/von Stutterheim/Carroll 2011). Die imperfektive Vergangenheitsform dagegen wurde gar nicht verwendet. Es scheint also, dass in Hier-undjetzt-Kontexten der Fokus auf der Abgeschlossenheit der dargebotenen Situation (und zwar unabhängig vom Situationstyp) im Fokus der tschechischen Sprecher steht.

Die Verteilung der Aspekt-Tempus-Formen in dem ostböhmischen Set ist auch für die übrigen Datensets repräsentativ mit der Einschränkung, dass die Differenz in der Verwendung der imperfektiven Simplex-Form im Vergleich zum sekundären Imperfektiv nicht in allen Sets statistisch signifikant ist. Obwohl die Unterschiede in der Formverteilung sehr interessant sind, kann darauf im Rahmen dieser Arbeit nicht näher eingegangen werden.

In der folgenden Abbildung 9 sind die Ergebnisse für die Verwendung der perfektiven Präsensform in Bezug auf die fünf Verbklassen dargestellt. ${ }^{68}$

68 Eine Übersichtstabelle mit absoluten Zahlen für alle Regionen und Verbklassen befindet sich im Anhang F, Tabelle 2. 


\section{Der Wahl der PfP-Form nach Verbklasse}

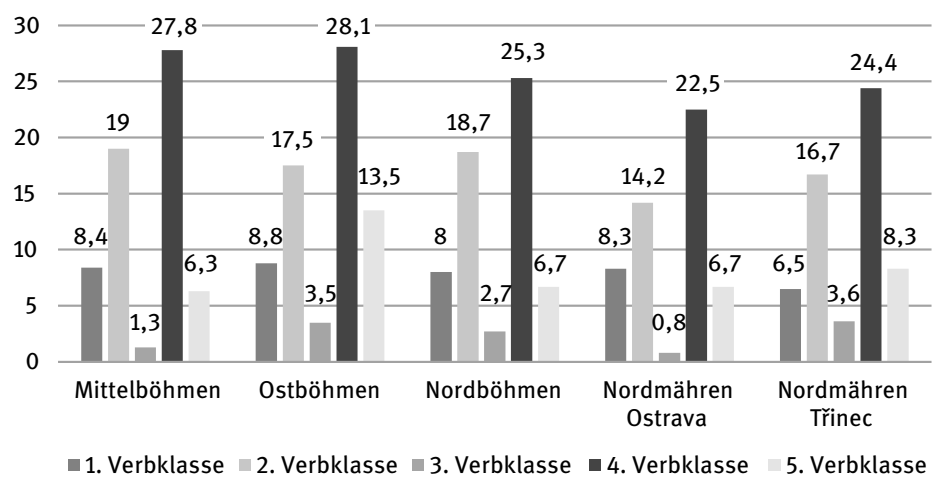

Abb. 9: Die Wahl der perfektiven Präsensform nach Verbklasse und Region

Die in Abbildung 9 präsentierten Daten belegen, dass die Wahl der perfektiven Präsensform mit der jeweiligen Verbklasse korreliert. Die größten Attraktoren sind Klasse II ( $\varnothing=17,2 \%)$ und Klasse IV ( $\emptyset=25,6 \%)$. Die Unterschiede zwischen den einzelnen Regionen in der Verwendung der PfP-Form in Klasse II sind statistisch nicht relevant: Mittelböhmen mit dem höchsten Prozentwert (19\%) gegenüber Nordmähren-Třinec mit dem niedrigsten Prozentwert (14,2\%) z = 1,35, ns. Das Gleiche trifft auch für Klasse IV zu: Ostböhmen mit dem höchsten Prozentwert $(28,1 \%)$ gegenüber Nordmähren-Ostrava mit dem niedrigsten Prozentwert $(22,5 \%) \mathrm{z}=1,09, n s$.

Die PfP-Form wird in Verbindung mit den Klassen I und III nicht so häufig gebraucht: Sie wird in der Klasse I in durchschnittlich nur 8\%, in der Klasse III in nur 2,3\% der Fälle gewählt. Auch hier sind die Schwankungen zwischen den Regionen statistisch nicht relevant: Klasse I Ostböhmen mit dem höchsten Prozentwert $(8,8 \%)$ gegenüber Nordmähren-Třinec mit dem niedrigsten Prozentwert $(6,5 \%)-z=0,8, n s ;$ Klasse III Ostböhmen mit dem höchsten Prozentwert (3,8\%) gegenüber Nordmähren-Ostrava mit dem niedrigsten Prozentwert $(0,8 \%) z=1,66$, $n s$. In der fünften Klasse kommt in den ostböhmischen Daten die PfP-Form viel häufiger vor als in den anderen Datensets: Ostböhmen mit dem höchsten Prozentwert $(13,5 \%)$ gegenüber Mittelböhmen mit dem niedrigsten Prozentwert $(6,3 \%) \mathrm{z}=12,1, p<.05$. Das Vorkommnis der PfP-Form in Verbindung mit der fünften Verbklasse ist in den anderen Regionen jedoch gleichmäßig.

Fragestellung 3: Hängt die Verwendung der PfP-Form mit Hier-und-jetzt-Interpretation von der Verbklasse ab, der das gegebene Verb angehört? 
Bezüglich der dritten Frage lässt sich festhalten, dass der Gebrauch der perfektiven Präsensform im Tschechischen von der Verbklasse abhängt. Am häufigsten kommt die PfP-Form im Zusammenhang mit Verben aus der II. und IV. Klasse vor, das niedrigste Vorkommnis wurde für die III. Klasse festgestellt. Es ist wichtig zu betonen, dass die Korrelation zwischen der jeweiligen Verbklasse und der Verwendung der PfP-Form in den verschiedenen Regionen vergleichbar ist. Die einzige Ausnahme stellt die Klasse V in den ostböhmischen Daten dar, in der signifikant mehr PfP-Formen vorkommen als in den übrigen Datensets.

Im Folgenden werden die tschechischen Daten mit den Daten aus dem Russischen verglichen. Dieser Gegenüberstellung liegt die Hypothese zugrunde, dass die perfektive Präsensform in den Hier-und-jetzt-Kontexten im Russischen keine Option darstellt. Abbildung 10 stellt die Ergebnisse des tschechisch-russischen Vergleichs dar:

Gebrauch der perfektiven Präsensform. Angaben in \%

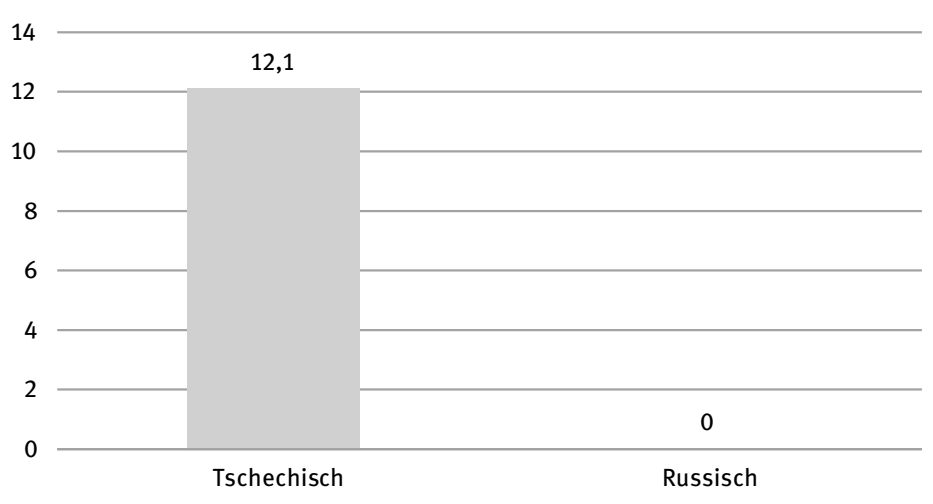

Abb. 10: Die Wahl der perfektiven Präsensform im Tschechischen ${ }^{69}$ und Russischen

Die russischen Daten zeigen, dass die Sprecher in den Hier-und-jetzt-Kontexten die perfektive Präsensform nie verwenden. Dies liegt darin begründet, dass sich die Semantik der PfP-Form im Russischen mit der Hier-und-jetzt-Bedeutung verträgt (vgl. z.B. Rathmayr 1976 oder eine beliebige Grammatik der Russischen). Das Ergebnis bestätigt darüber hinaus die vorliegenden Sprachproduktionsdaten (Schmiedtová/Sahonenko 2008). Ferner belegen die Befunde, dass die im Präfe-

69 Für die tschechischen Daten wurde für den vorliegenden Vergleich der Mittelwert berechnet. 
renztest verwendeten Szenarien tatsächlich einen Hier-und-jetzt-Kontext bei den Sprechern hervorgerufen haben. Mit anderen Worten hat sich der Präferenztest als Instrument zur Überprüfung der Kompatibilität der perfektiven Präsensform und Hier-und-jetzt-Interpretation bewährt.

\subsection{Fazit und Diskussion}

Die Studie hat gezeigt, dass Sprecher des Tschechischen die perfektive Präsensform wählen können, um auf Hier-und-jetzt-Kontexte Bezug zu nehmen. Und das, obwohl eine solche Verwendungsweise der PfP-Form von den Grammatiken des Tschechischen als nicht möglich beschrieben wird (vgl. Komárek et al. (Hgg.) 1986; Cvrček et al. (Hgg.) 2010).

Darüber hinaus wird deutlich, dass die Wahl der perfektiven Präsensform nicht etwa auf bestimmte Regionen beschränkt ist, sondern auf dem ganzen Gebiet Tschechiens - Böhmen, Mähren und Schlesien - vergleichbar häufig verwendet wird. Dabei ist hervorzuheben, dass die PfP-Form mit einer durchschnittlichen Verwendungshäufigkeit von 12,1\% die dritthäufigste Aspekt-Form darstellt. Diese Zahl belegt, dass der PfP-Form ein fester Status unter den Aspekt-Formen zuzuschreiben ist, die für den Hier-und-jetzt-Kontext als Option in Frage kommen.

Die Verwendung der perfektiven Präsensform mit Hier-und-jetzt-Bedeutung korreliert mit der Verbklasse. Die PfP-Form wird in Hier-und-jetzt-Kontexten am häufigsten mit Verben aus der II. und IV. Klasse, am seltensten mit Verben aus der III. Klasse kombiniert. Das hohe Vorkommnis der PfP-Form in der IV. Klasse könnte statistisch damit zusammenhängen, dass die meisten tschechischen Verben der IV. Klasse angehören (etwa 30\%, vgl. Cvrček et al. (Hgg.) 2010: 250 f.). Wenn wir annehmen, dass die perfektive Präsensform im tschechischen AspektTempus-System einen Sprachwandelprozess durchläuft bzw. durchlaufen ist, würde sich die IV. Klasse aufgrund ihrer Frequenz im sprachlichen Input sehr eignen, die perfektive Präsensform bei der Hier-und-jetzt-Bedeutung vor allen anderen zu integrieren (vgl. auch den sog. Kohäsionsparameter, Lehmann 1995). Für diese Hypothese spricht auch die Tatsache, dass die I. Klasse, die statistisch am geringsten gewichtet ist (etwa 7\%, vgl. Cvrček et al. (Hgg.) 2010: 250 f.), in dieser Studie zu den weniger starken Attraktoren der PfP-Form zählt.

Auch hier hat die Datenanalyse gezeigt, dass die Korrelation zwischen Verbklasse und Häufigkeit der Verwendung der PfP-Form nicht regional oder dialektal gebunden ist - die Affinität bestimmter Verbklassen, die PfP-Form mehr oder weniger stark anzuziehen, ist in allen Regionen gleich ausgeprägt. Die einzige Ausnahme stellt die übermäßig häufige Verwendung der PfP-Form mit Verben 
aus der V. Klasse in den ostböhmischen Daten dar. Eine Erklärung für diesen Befund steht noch aus, es ist aber beabsichtigt, die Auswirkung der Verbklassen auf die Verwendung der PfP-Form im Tschechischen in weiteren Arbeiten näher zu untersuchen.

Im Kontext der Verbklassen stellt sich darüber hinaus die Frage, inwiefern die Einteilung in verschiedene Konjugationsklassen vom grammatischen Aspekt, möglicherweise aber auch von der Semantik der Verben abhängig ist. Zu den beiden potenziellen Zusammenhängen liegen aktuell jedoch noch keine Daten vor. In Bezug auf eine solche Studie wäre interessant, herauszufinden, ob die beobachtete Korrelation zwischen der Häufigkeit der Verwendung der PfP-Form und den Konjugationsklassen auch mit den aspektuellen Eigenschaften der Verbklassen in Verbindung stehen könnte.

Die Daten aus dem Russischen bekräftigen die Unterschiede zwischen dem Tschechischen und dem Russischen hinsichtlich der Kombinierbarkeit der PfPForm mit der Hier-und-jetzt-Bedeutung: Im Russischen ist diese Kombination für den besagten Kontext nicht zulässig. Dieser Befund ist im Einklang mit den Ergebnissen aus Sprachproduktionsdaten, in denen die russischen Probanden die PfP-Form ebenfalls nie verwendet haben, die tschechischen Sprecher aber sehr wohl (Schmiedtová/Sahonenko 2008: 58 ff.). ${ }^{70}$

Die Unterschiede in der Verwendung der präfigierten perfektiven Formen im Tschechischen und im Russischen lassen sich auch für längere narrative Texte dokumentieren. Die Daten aus den Nacherzählungen des Quest-Filmes ${ }^{71}$ zeigen,

70 In dem Szenario Nr. 34 wurde ein für beide Systeme relativ neues Lexem getestet. Es handelte sich um das aus dem Englischen entlehnte Verb klikat Impf / poklinknout Perf ('klicken') in einem Kontext, in dem Ware aus dem Internet per Mausklick bestellt werden. Das Verb wird in beiden Sprachen gebraucht, es zeigt jedoch unterschiedliche Tendenzen bezüglich seiner Kombinierbarkeit mit den verschiedenen Aspekt-Tempus-Formen auf: Während in den russischen Daten dieses Verb ausschließlich mit der imperfektiven Simplex-Form im Präsens $(88,6 \%)$ oder in der Vergangenheit $(8,6 \%)$ sowie in Verbindung mit dem sekundären Imperfektiv $(2,9 \%)$ vorkommt, wird es im Tschechischen nicht nur mit den beiden imperfektiven Simplex-Formen (Präsens durchschnittlich 53,9\%, Vergangenheit durchschnittlich 8,22\%) sondern auch mit der perfektiven Präsensform (durchschnittlich 21,7\%) sowie der perfektiven Vergangenheitsform (durchschnittlich 7\%) kombiniert. Diese Tendenzen lassen vermuten, dass der Prozess der Lexikalisierung eines neuen Wortes direkt von den verfügbaren Aspekt-Formen abhängig ist und somit im Tschechischen anders als im Russischen verläuft.

71 Der Film Quest wurde 1996 von Tyron Montgomery und Thomas Stellmach produziert und gedreht. Es handelt sich um die Geschichte einer Tonfigur, die auf der Suche nach Wasser ist. Dabei durchwandert die Figur verschiedene Welten (z.B. Papier- oder Steinwelt), in denen sie unterschiedlichen Gefahren entkommen muss. Im Jahr 1997 wurde Quest mit einem Oscar für den besten Zeichentrickfilm ausgezeichnet. 
dass russische Sprecher perfektive Formen ausschließlich in der Vergangenheit verwenden (perfektive Vergangenheitsform). ${ }^{72}$ Die perfektive Präsensform wird von den russischen Sprechern in den Daten dieser Studie weder in Verbindung mit Präsens- oder Futurbedeutung noch in Form des so genannten historischen (oder narrativen) Präsens ${ }^{73}$ verwendet. Das Bild im Tschechischen hingegen stellt sich anders dar: In den Quest-Nacherzählungen kommt die perfektive Präsensform relativ häufig vor, z.B. in der zweiten Episode dieses Filmes liegt der Anteil der perfektiven Präsensform für Äußerungen im Vordergrund bei etwa $61 \%$ (68 von insgesamt 112 Vorkommnissen). ${ }^{74}$ Wie lassen sich diese Unterschiede interpretieren?

Zunächst ist zu betonen, dass die Quest-Daten unter Verwendung eines anderen Designs erhoben wurden, als es den bisher betrachteten Sprachproduktionsund Präferenztestdaten zugrunde liegt. Die Sprachproduktions- und Präferenztestdaten wurden in einem Online-Design erhoben: Die Sprecher haben die Stimuli versprachlicht, während sie ihnen präsentiert wurden. Mit anderen Worten, es gab einen direkten Bezug zum deiktischen Jetzt. Alle Quest-Daten wurden dagegen in einem Offline-Verfahren erhoben. Das heißt, die Probanden haben den Film zuerst am Stück gesehen, dann wurde der Film sofort nach jeder Episode gestoppt und die Probanden aufgefordert, die jeweilige Episode nachzuerzählen. Der kontextuelle Bezugsrahmen für die Nacherzählungen lag in der Vergangenheit, da die zu beschreibenden Situationen bereits geschehen waren. Dieses Design eröffnet also die Möglichkeit, die PfP-Form als historisches Präsens zu verwenden.

Auch bezüglich des historischen Präsens gibt es zwischen dem Russischen und dem Tschechischen wichtige Unterschiede. Dickey (2011: 175) vertritt die Meinung, dass das Russische in Folge von diachronen Änderungen des aspektuellen Systems, die im Rahmen der Abgrenzung der ost- und westslawischen Sprachen stattfanden, die Verwendung der perfektiven Formen im historischen Präsens ganz verloren hat. In der empirisch gestützten Untersuchung von Rathmayr (1976: 128) werden dagegen wenige Belege für die Verwendung der perfektiven Präsensform als historisches Präsens präsentiert. In Anlehnung an die vorgenannte Literatur zum Thema (Rathmayr 1976; Dickey 2011) sowie die hier präsentierten Daten kann festgehalten werden, dass im Russischen die Möglichkeit der Verwendung der perfektiven Präsensform als historisches Präsens ein-

72 Vgl. Sahonenko (2004: 55-58).

73 Historisches Präsens wird verwendet, um auf vergangene Ereignisse Bezug zu nehmen. Man bezeichnet es auch als das Präsens der belebten Erzählung (vgl. Isačenko 1982; Panzer 1991).

74 Vgl. Placzková (2009: 85). 
geschränkt bzw. sehr selten möglich ist. Im Gegensatz dazu wird für narrative Texte im Tschechischen angenommen, dass neben der imperfektiven auch die perfektive Präsensform zulässig ist (vgl. Berger 2010; Dickey 2011). In der kürzlich erschienenen korpusbasierten Grammatik des Tschechischen werden in Bezug auf das historische Präsens aber nur Belege mit imperfektiven Formen erwähnt. ${ }^{75}$ Zum Beispiel: tsch. Tak třeba včera mi povídá - dt. So beispielsweise gestern sagt er mir (Cvrček et al. (Hgg.) 2010: 240). Zum Vergleich ein Beispiel aus den tschechischen Quest-Daten. ${ }^{76}$ Der Sprecher in (19) verwendet durchgehend die perfektive Präsensform:

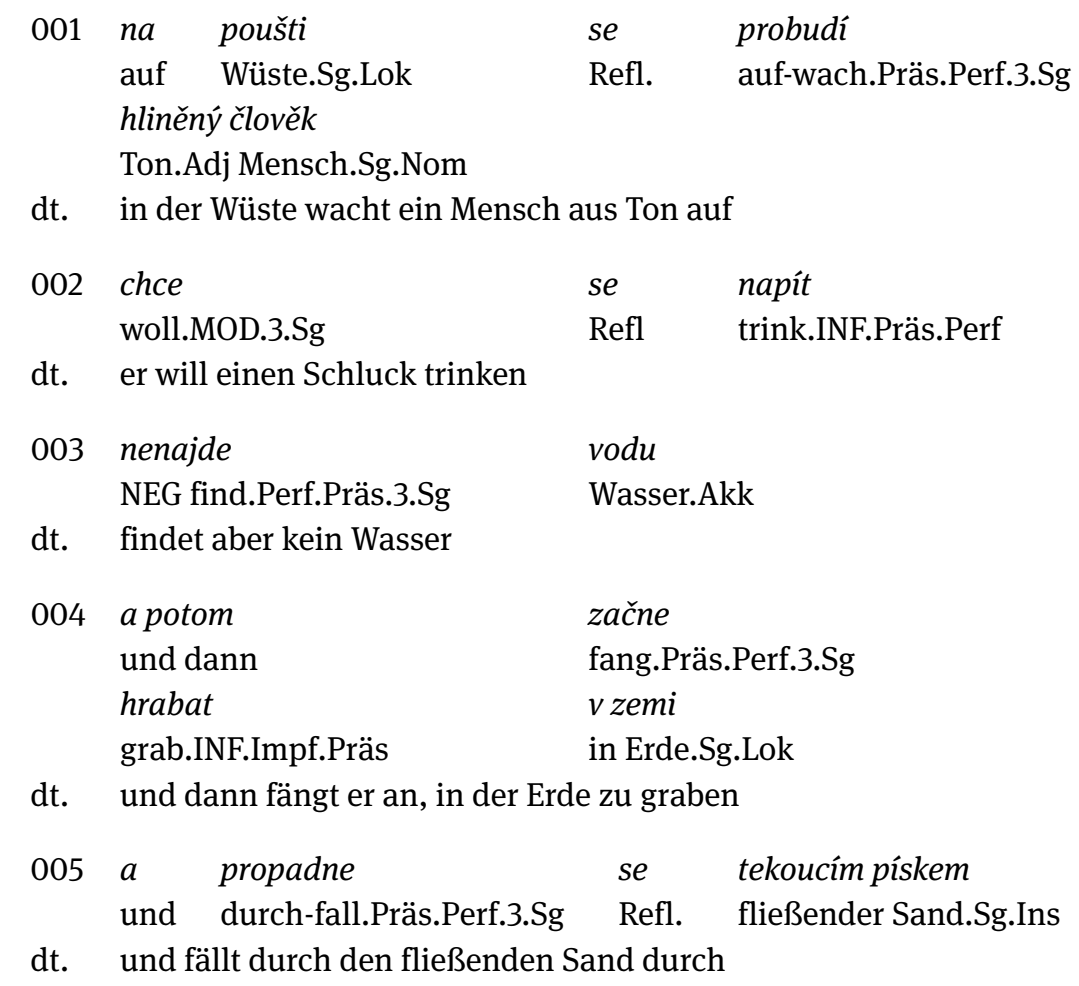

75 Eine ältere Grammatik aus dem Jahre 1986 nimmt an, dass im Zusammenhang mit historischem Präsens auch perfektive Präsensformen verwendet werden können (Komárek et al. (Hgg.) 1986: 179).

76 Vielen Dank an Frau Andrea Germann für die Möglichkeit, ihre Daten zum Tschechischen verwenden zu dürfen. 
Es stellt sich die Frage, ob die in den tschechischen narrativen Texten häufig verwendeten perfektiven Präsensformen zwingend als historisches (narratives) Präsens interpretiert werden müssen. Die hier präsentierten Daten legen die Vermutung nah, dass die PfP-Form möglicherweise auch in Nacherzählungen mit der Hier-und-jetztBedeutung verbunden werden kann. Dies ist jedoch eine Fragestellung, die in weiteren experimentellen und korpusbasierten Arbeiten zu untersuchen ist.

Zugleich sollte aber betont werden, dass die temporale Interpretation dieser Form von dem zugrundeliegenden temporalen Bezugsrahmen abhängig ist und sich dieser eher unter experimentellen Bedingungen kontrollieren als unter Verwendung von Korpusdaten vorgeben und kontrollieren lässt. Aus den Korpusdaten lässt sich primär auf Häufigkeiten und Korrelationen bzw. Kollokationen schließen. Unter diesem Gesichtspunkt ist die Angabe in der korpusbasierten CvrčekGrammatik (Cvrček et al. (Hgg.) 2010) sehr relevant: Dort werden nämlich nur die imperfektiven Formen für die Verwendung des historischen Präsens erwähnt.

Die hier dargestellte Datenlage zeigt, dass die perfektive Präsensform im Tschechischen ein breiteres Bedeutungsspektrum bedient als die russische Präsensform: Im Tschechischen kann sich die PfP-Form nicht nur auf die Zukunft, sondern auch auf die Gegenwart beziehen. Diese besondere Verwendung der PfPForm lässt sich zum einen mit der von Dickey (2000) vorgeschlagenen Abgrenzung der westslawischen (z.B. Tschechisch, Slowakisch, Sorbisch, Slowenisch) und ostslawischen (z.B. Russisch, Bulgarisch) Sprachen zusammenbringen: In den westslawischen Sprachen ist die Wahl einer aspektuellen Form weniger eingeschränkt als in der ostslawischen Gruppe. In der westslawischen Gruppe ergeben sich also viel mehr Kontexte, für die nicht eine bestimmte Aspektwahl zwingend ist, sondern aus mehreren aspektuellen Möglichkeiten gewählt werden kann (vgl. Berger 2011).

Zum anderen erscheint es nicht unplausibel, dass die Verwendung der perfektiven Präsensform mit Hier-und-jetzt-Bedeutung auf den lang andauernden Sprachkontakt zum Deutschen zurückzuführen ist: Präfigierte telische Verben sind mit der Hier-und-jetzt-Bedeutung im Deutschen ohne Weiteres kombinierbar (dt. Hans isst gerade/jetzt/in diesem Moment einen Apfel auf). Neue psycholinguistische Studien zur Versprachlichung und Konzeptualisierung von Einzelereignissen (siehe Kap. 5; vgl. Schmiedtová/von Stutterheim/Carroll 2011) sowie diachron orientierte Arbeiten (vgl. Dickey 2011) lassen vermuten, dass der Kontakt zum Deutschen einen großen Einfluss auf die Sprachsysteme der westslawischen Sprachen ausgeübt hat. Für das Tschechische könnte dies in besonderem Maße zutreffen (siehe auch Kap. 4).

Abschließend soll Fragestellung 4 diskutiert werden: Welche Implikationen haben die Befunde über die Verwendung der PfP-Form mit Hier-und-jetzt-Bedeutung für das Aspektsystem des Tschechischen? 
Die erste Konsequenz ist die Erweiterung des tschechischen Aspektsystems: In Hier-und-jetzt-Kontexten kann neben der imperfektiven Simplex-Form und der sekundären imperfektiven Form zusätzlich auch die PfP-Form gewählt werden. In weiteren Arbeiten soll untersucht werden, welche Faktoren die Wahl einer bestimmten Aspektform bei Hier-und-jetzt-Kontexten beeinflussen. Vermutlich spielen dabei der Situationstyp bzw. die Proximität der Zustandsveränderung (das Eintreten des Nachzustands) sowie der Sprachmodus (mündlich vs. schriftlich) eine wichtige Rolle. In Bezug auf den Sprachmodus ist anzumerken, dass die Präferenzen im mündlichen Modus viel ausgeprägter sind als im schriftlichen: Tschechische Sprecher verwenden in mündlicher Produktion die PfP-Formen mit Hier-und-jetzt-Bedeutung viel häufiger als sekundäre Imperfektiva oder imperfektive Simplex-Formen (vgl. Berger 2011; Schmiedtová/von Stutterheim/Carroll 2011; Kap. 8).

Eine zweite Implikation betrifft die Veränderung der Kategorie Perfektivität. Die Befunde dieser Studie zeigen, dass die perfektive Präsensform im Tschechischen mehrdeutig ist. Die PfP-Form hat nicht nur Futurbedeutung (vgl. Schmiedtová 2003a, 2003b; Cvrček et al. (Hgg.) 2010; Schmiedtová/von Stutterheim/Carroll 2011), sondern kann auch mit Hier-und-jetzt-Bedeutung verwendet werden. Für diese Bedeutung gilt, dass das Eintreten eines Nachzustands assertiert wird, obwohl dieser faktisch gar nicht oder noch nicht eingetreten ist. Mit anderen Worten, der Nachzustand muss unter der Hier-und-jetzt-Interpretation per default inferiert werden. Im Unterschied zur Verwendung der perfektiven Form in der Vergangenheit ${ }^{77}$ wird durch die perfektive Präsensform mit Hier-und-jetzt-Bedeutung keine Faktizität bezeugt, sondern vielmehr die 'Einschätzung' der Wahrscheinlichkeit, mit der ein Nachzustand eintritt, miteinbezogen.

Aufgrund dieser temporal-semantischen Merkmale ist die PfP-Form mit Hierund-jetzt-Bedeutung kompatibel mit der sogenannten holistischen Perspektive, die im Tschechischen für die Konzeptualisierung und Verbalisierung unterschiedlicher Ereignistypen bevorzugt gewählt wird (siehe Kap. 5-9). Unter dieser Perspektive wird stets auf das Gesamtereignis Bezug genommen. Das heißt, dass beispielsweise bei Ereignissen mit Zustandsveränderung der Nachzustand, bei Bewegungsereignissen dagegen das Ziel (Endpunkt) der Bewegung inferiert wird.

Die Frage nach dem Verhältnis zwischen der gewählten Konzeptualisierung einerseits und den Veränderungen im tschechischen Aspektsystem andererseits lässt sich anhand der zur Verfügung stehenden Daten nicht abschließend beant-

77 Für die perfektive Vergangenheitsform gilt, dass die Sprechzeit und der Nachzustand entweder zusammenfallen, oder der Nachzustand vor der Sprechzeit eingetreten sein muss: tsch. Právě/Včera to dopsala - dt. Gerade/Gestern hat sie es fertig schreiben. 
worten. Dennoch ist es naheliegend, dass die zugrundeliegende Konzeptualisierung, die sogenannte holistische Perspektive, zu den hier dargestellten Veränderungen der PfP-Form geführt oder zumindest diese begünstig haben und die Ausbildung der holistischen Perspektive im Tschechischen auf den sprachlichen Einfluss des Deutschen zurückzuführen ist.

\subsection{Zusammenfassung}

In diesem Kapitel wurde eine sehr umfangreiche, auf Präferenzurteilen basierte Studie zur Verwendung der perfektiven Präsensform (PfP-Form) im Tschechischen vorgestellt. Im Wesentlichen zeigen die Befunde, dass die Anwendungsdomäne der PfP-Form sich stark geändert hat. Anders als in den tschechischen Grammatiken dargestellt, wird die PfP-Form im heutigen Tschechisch auch unter dem Einbezug der deiktischen Hier-und-jetzt-Bedeutung verwendet. Diese Verwendung ist weder von der Verbalklasse noch der regionalen Zugehörigkeit der Probanden abhängig. Diese Ergebnisse werden als Folge des Sprachkontakts zwischen dem Deutschen und Tschechischen interpretiert. 